\title{
Desarrollo e implementación de una vía clínica a los pacientes que inician la hemodiálisis de forma programada
}

\author{
Moisés Navarro Bermúdez
}

\section{Enfermero}

Licenciado en antropología social y cultural

Servicio de Nefrología. Hospital de Jerez. Cádiz.

\section{Resumen}

Una vía clínica pretende ser un instrumento institucionalizado, donde todos los profesionales del servicio sepan en todo momento todas las actividades y acciones que tienen que llevar a cabo el tiempo que dura esta actividad asistencial y los usuarios/familiares conozcan desde el primer momento toda la información de la asistencia que se les va a prestar.

Con el fin de diseñar una vía clínica se formó un grupo de trabajo interdisciplinar que elaboró un instrumento compuesto por:

- Una matriz temporal, donde se detallan por día las actividades de todos los profesionales implicados en la vía clínica, y se especifican otros aspectos importantes como son las pruebas complementarias, dieta y medicación.

- Hoja de variaciones. Donde se detallan tanto las desviaciones de la vía como las acciones realizadas o su justificación.

- Hoja de información al paciente y/o familiar. Incluye una serie de ilustraciones que informan al paciente sobre la evolución más frecuente del

\begin{tabular}{|c|}
\hline Correspondencia: \\
Moisés Navarro Bermúdez \\
c/ Rodrigo de Bastidas $12,2^{\circ} \mathrm{A}$ \\
11500 Pto Sta Maria (Cádiz) \\
monavber@ono.com \\
\hline
\end{tabular}

proceso. Es una versión gráfica simplificada de la matriz para una fácil comprensión del paciente.

- Encuesta de satisfacción del paciente y/o familiar. Se entrega al paciente para que exprese de forma anónima su opinión sobre la atención y cuidados prestados. Engloba preguntas respecto a la información recibida, la atención, aspectos de hostelería, etcétera.

Hemos considerado todo el equipo, como periodo de reevaluación de la misma cuando alcancemos los 10 pacientes, para poder realizar modificaciones 0 áreas de mejoras de la misma.

\section{Development and implementation of a clinical pathway for patients starting programmed haemodialysis}


at all times the activities and actions they have to carry out, the time this care activity lasts, and users and relatives know right from the start all the information on the care they will be given.

In order to design a clinical pathway, a multidisciplinary working group was formed which drew up an instrument made up of:

- A time chart detailing by day the activities of all the professionals involved in the clinical pathway, and specifying other important aspects such as complementary tests, diet and medication.

-Variance sheet. Where both deviations from the pathway and the actions realized or their justification are detailed.

- Information sheet for the patient and/or relative. It includes a series of illustrations that inform the patient of the most common evolution of the process. It is a simplified graphic version of the time chart so that it can be easily understood by the patient.

- Patient and/or relative satisfaction survey. This is given to the patient so that he or she can give his or her opinion anonymously on the care received. It includes questions on the information received, care, catering aspects, etc.

We have considered the entire team, and reassessed the pathway when we reached 10 patients, in order to make modifications or determine areas for improvement.

\section{KEY WORDS:}

\section{- CLINICAL PATHWAYS}

- DIALYSIS

- CARE QUALITY

- CARE

- CHRONIC RENAL INSUFFICIENCY

\section{Introducción}

El Hospital de Jerez de la Frontera (Cádiz), atiende al área norte de la provincia de Cádiz, aproximadamente a unos 300.000 habitantes, y en concreto, la unidad de hemodiálisis de este hospital atiende a más de 200 pacientes al año. Nuestro servicio de Nefrología además tiene tres centros periféricos concertados de hemodiálisis que dependen de nosotros, uno en Jerez, otro en Sanlúcar de Barrameda y otro, recientemente inaugurado, en Arcos de la Frontera.

En el servicio de Nefrología inician actualmente la diálisis unos 50 pacientes al año, pacientes con grandes demandas sanitarias y con gran desconocimiento sobre lo que este nuevo proceso supone en sus vidas, tanto para el paciente como para su familia.

Según indica Hernández et $\mathrm{al}^{1}$, en los últimos años venimos asistiendo a una serie de cambios en el sector sanitario que afectan a pacientes, profesionales y gestores. Los recursos limitados y un interés creciente por garantizar la calidad de la asistencia sanitaria han favorecido la aparición, desarrollo e implantación de métodos de práctica óptima, utilizando los principios de la medicina basada en la evidencia, aplicados a la gestión de la enfermedad o el proceso.

Para poder dar respuesta a estos usuarios y familiares y poder ofrecer una asistencia de calidad han surgido las vías clínicas. Entendiéndose éstas, como un sistema de estandarización elaborada por profesionales sanitarios como forma de adaptar las guías clínicas a la práctica

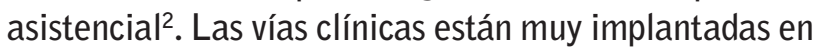
los EE.UU. En nuestro país ya existe cierta experiencia en el desarrollo e implantación de vías clínicas.

La Organización Mundial de la Salud define la calidad de la asistencia sanitaria como la garantía de que los pacientes reciben el conjunto de servicios diagnósticos y terapéuticos más adecuados para conseguir unos resultados óptimos, con el mínimo riesgo de efectos iatrogénicos y la mayor satisfacción posible del usuario con el proceso.

Tal como indica Viana et $\mathrm{al}^{3}$, en estos últimos años hemos asistido a un interés creciente por garantizar una calidad óptima de la asistencia sanitaria, disminuyendo la variabilidad de la práctica clínica, con el objetivo de mejorar la calidad asistencial. Las vías clínicas son 
una de las posibles soluciones a la variabilidad de la práctica clínica. Se definen como planes asistenciales que se aplican a enfermos con determinada patología y que presentan un curso clínico predecible. Constituyen una herramienta de mejora de la calidad que tiene el objetivo de mejorar el trabajo multidisciplinario en equipo, integrando las guías y protocolos que han sido introducidos en la práctica clínica.

Nuestra comunidad autónoma (Andalucía), no se mantiene ajena a estas necesidades, ya que a través del III Plan Andaluz de Salud ${ }^{4}$ plantea en una de sus cuatro metas la "Atención centrada en el ciudadano", pretendiendo que éstos tengan la mejor opinión sobre su funcionamiento, asegurando los mejores resultados, un enfoque de equidad, prestaciones óptimas y eficiencia en el empleo de los recursos.

Es un aspecto prominente en el grado de satisfacción del usuario tener en cuenta su opinión, percepciones y sentimientos haciéndole partícipe, colaborador de su enfermedad; por ello, dar respuesta a esa demanda constituye un aspecto clave de la calidad percibida. Desde hace tiempo se reconoce la importancia de la satisfacción del paciente como medida de resultado 5 .

Además, el Plan de Calidad de la Consejería de salud ${ }^{5}$, establece la necesidad de adoptar ${ }^{5}$ "...... un enfoque de calidad centrado en el elemento básico del sistema sanitario: el proceso asistencial, integral y único, entendido desde la perspectiva del ciudadano. Así, la Continuidad Asistencial pasa a ser el criterio clave y determinante que inspira organización, funcionalidad, formas de trabajo, formación de profesionales, etc."

\section{Objetivos}

El objetivo general que nos planteamos principalmente fue:

- Desarrollo e implementación de una vía clínica al inicio de la hemodiálisis programada.

Mientras que los objetivos específicos del mismo fueron:

- Diseño de la matriz de la vía clínica para la atención de los pacientes que inicien la hemodiálisis programada en el servicio de nefrología del Hospital de Jerez ${ }^{6-11}$.
- Diseño de la hoja de información para el paciente/ familia que se incluya en la vía clínica.

- Implementación de la vía clínica en el Servicio de Nefrología/hemodiálisis del Hospital de Jerez.

\section{Metodología}

El desarrollo de la vía clínica se desarrolló en cuatro etapas:

\section{$1^{\text {a Etapa: }}$}

Se establece el compromiso de desarrollar una vía clínica para el "inicio de la hemodiálisis programada" y se crea un grupo de trabajo con personal médico y de enfermería del Servicio de Nefrología/Hemodiálisis del Hospital de Jerez. Se revisó la bibliografía existente relativa a diferentes aspectos relacionados con la hemodiálisis y el inicio de los pacientes en la misma, con el objeto de buscar la mejor evidencia disponible para cada uno de ellos.

Se designó al Líder de la vía clínica, que recayó en el supervisor de nefrología y se constituyó el grupo de trabajo. El grupo de trabajo fue multidisciplinar y estuvo formado por un médico, cuatro enfermeros y una auxiliar de enfermería.

\section{$2^{\mathrm{a}}$ Etapa}

Se procedió al diseño de la matriz de la vía clínica y de la hoja de información al paciente/familiar. Se planificó la secuencia de procedimientos médicos, de enfermería y administrativos, así como la responsabilidad de cada profesional.

Se especificaron las áreas de incertidumbre y dudas sobre determinadas prácticas (adecuación y seguridad) y se procedió a una búsqueda y revisión bibliográfica para seleccionar la mejor práctica utilizando la sistemática de la Asistencia Basada en la Evidencia (ASBE).

Se establecieron acuerdos, si fuera necesario, con otras unidades de soporte. Por ejemplo: pruebas diagnósticas y se analizaron posibles puntos críticos para prevenir Errores, por ejemplo: identificación del paciente. 
Se buscó la integración de mapas de cuidados estandarizados:

- Identificación de los principales problemas-diagnósticos enfermeros relacionados con este tipo de pacientes atendidos en la unidad.

- Criterios de resultados: Objetivos a alcanzar para la mejora de esos problemas.

- Indicadores que se ponen en marcha cada día para lograr los resultados esperados (NOC).

Con respecto a la Hoja de Información al paciente/ familia el grupo debió:

- Determinar los puntos clave de información (acogida, alta, su médico es...).

- Dará una información sencilla y apoyada con dibujos.

- Establecerá el "Procedimiento de Consentimiento Informado".

Se diseñó un borrador de la matriz temporal, así como del resto de documentos de la vía: hojas de tratamiento, variaciones, encuesta de satisfacción del paciente y/o familiares, hoja de información para el paciente y/o familiares e indicadores de evaluación.

Se presentó el borrador al conjunto de profesionales implicados para su discusión y modificación si fuera preciso.

\section{$3^{\text {a Etapa: }}$}

Se procedió a la presentación de la vía clínica con el resto de los profesionales implicados y a la posterior implementación de la misma en el servicio/unidad.

\section{$4^{\mathrm{a}}$ Etapa}

Establecemos realizar un análisis de la misma cuando tratemos a los 10 primeros pacientes, para valorar posibles áreas de mejora y llevar a cabo modificaciones de la misma si fuera pertinente.

\section{Sujetos y ámbitos de estudio}

Los sujetos de estudio serán todos los pacientes que inicien la hemodiálisis de forma programada en el servicio de nefrología/diálisis del Hospital de Jerez de la Frontera (Cádiz).

\section{Criterios de inclusión}

Pacientes que inicien la hemodiálisis de forma programada, que estén incluidos en consulta de ERCA y que tengan acceso vascular definitivo.

\section{Resultados}

En definitiva los documentos que integran la vía clínica es:

- Hoja matriz de la vía clínica (tabla 1). Donde se detallan por día las actividades de todos los profesionales implicados en la vía clínica, y se especifican otros aspectos importantes como son las pruebas complementarias, dieta y medicación.

- Hoja de variaciones (tabla 2). Donde se detallan tanto las desviaciones de la vía como las acciones realizadas o su justificación.

- Hoja de información al paciente y/o familiar (tabla 3). Incluye una serie de ilustraciones que informan al paciente sobre la evolución más frecuente del proceso. Es una versión gráfica simplificada de la matriz para una fácil comprensión del paciente.

- Encuesta de satisfacción del paciente y/o familiar. Se entrega al paciente para que exprese de forma anónima su opinión sobre la atención y cuidados prestados. Engloba preguntas respecto a la información recibida, la atención, aspectos de hostelería, etcétera.

\section{Discusión/Conclusiones}

Para evaluar los posibles cambios o modificaciones de esta vía clínica, hemos decidido realizar una autoevaluación después de atender los diez primeros pacientes, para así poder analizar posibles áreas de mejora, que nos ayuden a dar a nuestros ciudadanos una asistencia de calidad. 
[ M. Navarro Bermúdez ]

Desarrollo e implementación de una vía clínica de los pacientes que inician la hemodiálisis de forma programada

\begin{tabular}{|c|c|c|c|c|}
\hline & DÍA 0 & $\begin{array}{l}\text { DÍA } 1 \\
1^{\text {a }} \text { diálisis }\end{array}$ & $\begin{array}{l}\text { DÍA } 2 \\
2^{\mathrm{a}} \text { diálisis }\end{array}$ & $\begin{array}{l}\text { DÍA } 3 \\
3^{\text {a }} \text { diálisis }\end{array}$ \\
\hline $\begin{array}{l}\text { CIRCUITO } \\
\text { DE CONTACTO }\end{array}$ & $\begin{array}{l}\text { El nefrólogo contacta con } \\
\text { la unidad de agudos (Ext } \\
\text { 099) para programación de } \\
\text { paciente }\end{array}$ & & & \\
\hline ACTUACIÓN MÉDICA & $\begin{array}{l}\text { Confirmación de cita. En- } \\
\text { trega de hoja de informa- } \\
\text { ción al paciente }\end{array}$ & $\begin{array}{l}\text { HC } \\
\text { Exploración física } \\
\text { Petición de pruebas }\end{array}$ & $\begin{array}{l}\text { Se evaluará al paciente } \\
\text { sólo si se producen inci- } \\
\text { dencias }\end{array}$ & $\begin{array}{l}\text { Exploración clínica. } \\
\text { Elaboración de informe de } \\
\text { alta. }\end{array}$ \\
\hline $\begin{array}{l}\text { CUIDADOS } \\
\text { DE ENFERMERÍA }\end{array}$ & $\begin{array}{l}\text { El enfermero le entregará } \\
\text { la hoja de información al } \\
\text { paciente/familia. }\end{array}$ & $\begin{array}{l}\text { Protocolo de acogida del } \\
\text { paciente en HD: } \\
\text { 1. Breve descripción de la } \\
\text { diálisis. } \\
\text { 2. Se le mostrará la unidad. } \\
\text { 3. Conceptos básicos de } \\
\text { FAV. } \\
\text { 4. Conceptos básicos sobre } \\
\text { peso, dieta y restricción } \\
\text { de líquidos. } \\
\text { Se le facilitará una } \\
\text { "Guía para pacientes con } \\
\text { tratamiento de HD". } \\
\text { Se le realizará valoración } \\
\text { enfermera, escalas } \\
\text { clinimétricas, se abrirá plan } \\
\text { de cuidados e historia de } \\
\text { diálisis. } \\
\text { Duración de tratamiento } \\
\text { (1ª sesión): } \\
2 \text { h de HD. Sin pérdidas. } \\
\text { Perfusión continua de } \\
\text { Manitol } 20 \% \text {. Dializador } \\
\text { de baja permeabilidad } \\
\text { Heparina Clexane } 20\end{array}$ & $\begin{array}{l}\text { Valoración del paciente: } \\
\text { 1.Toma de constantes } \\
\text { 2. Comprobación de la } \\
\text { permeabilidad de la FAV. } \\
\text { 3. Preparación para el } \\
\text { tratamiento. } \\
\text { Seguimiento del Plan de } \\
\text { cuidados. } \\
\text { EPS ( } 1^{a} \text { sesión): dieta y } \\
\text { hábitos saludables. } \\
\text { Duración de tratamiento } \\
\text { (2ª sesión): } \\
2 \text { h de HD. Sin pérdidas. } \\
\text { Perfusión continua de } \\
\text { Manitol } 20 \% \text {. Dializador } \\
\text { de baja permeabilidad } \\
\text { Heparina Clexane } 20\end{array}$ & $\begin{array}{l}\text { Valoración del paciente: } \\
\text { 1. Toma de constantes } \\
\text { 2. Comprobación de la } \\
\text { permeabilidad de la FAV. } \\
\text { 3. Preparación para el } \\
\text { tratamiento. } \\
\text { Seguimiento del Plan de } \\
\text { cuidados. } \\
\text { EPS ( } 2^{\text {a }} \text { sesión): Cuidados } \\
\text { FAVI } \\
\text { Duración de tratamiento } \\
\text { (3a sesión): } \\
2 \text { h } 30 \text { minutos de HD. Sin } \\
\text { pérdidas. } \\
\text { Perfusión continua de } \\
\text { Manitol } 20 \% \text {. Dializador } \\
\text { de baja permeabilidad } \\
\text { Heparina Clexane } 20 \\
\text { Elaboración de ICC. } \\
\text { Aportación al paciente/ } \\
\text { cuidador de encuesta de } \\
\text { satisfacción. }\end{array}$ \\
\hline $\begin{array}{l}\text { AUXILIAR } \\
\text { ADMINISTRATIVO }\end{array}$ & Gestión de ambulancias & & & $\begin{array}{l}\text { Gestión de traslados. } \\
\text { Gestión de Alta y } \\
\text { derivación a Centro } \\
\text { periférico de diálisis o a la } \\
\text { unidad de diálisis crónica. }\end{array}$ \\
\hline $\begin{array}{l}\text { AUXILIAR DE } \\
\text { ENFERMERÍA }\end{array}$ & $\begin{array}{l}\text { Tramitación y programa- } \\
\text { ción de inicio de paciente } \\
\text { en diálisis }\end{array}$ & $\begin{array}{l}\text { Entregará documento al } \\
\text { paciente/familia donde se } \\
\text { indique turno de diálisis. }\end{array}$ & $\begin{array}{l}\text { Contactará con centro } \\
\text { periférico para tramitación } \\
\text { de derivación del paciente. }\end{array}$ & $\begin{array}{l}\text { Confirmará con centro } \\
\text { periférico o unidad de } \\
\text { crónicos el turno asignado } \\
\text { al paciente. }\end{array}$ \\
\hline $\begin{array}{l}\text { ESTUDIOS } \\
\text { COMPLEMENTARIOS }\end{array}$ & & $\begin{array}{l}\text { Analítica general. } \\
\text { Marcadores (VIH, HB y } \\
\text { HC) } \\
\text { Rx torax, simple y lateral de } \\
\text { abdomen y ECG }\end{array}$ & BQ post & $\begin{array}{l}\text { Hemograma } \\
\text { BQ post }\end{array}$ \\
\hline \multicolumn{5}{|l|}{ MEDICACIÓN } \\
\hline DIETA & & $\begin{array}{l}\text { No tomar nada durante la } \\
\text { diálisis }\end{array}$ & $\begin{array}{l}\text { No tomar nada durante la } \\
\text { diálisis }\end{array}$ & $\begin{array}{l}\text { No tomar nada durante la } \\
\text { diálisis }\end{array}$ \\
\hline
\end{tabular}

Tabla 1. Matriz de la vía clínica de inicio en hemodiálisis 


\section{ETIQUETA}

(En su defecto, indique nombre y ubicación del paciente)

NOMBRE:

CAMA:

Fecha $1^{a}$ DIÁLISIS Fecha de alta

Diagnostico.

\section{SERVICIO DE HEMODIÁLISIS.}

VARIACIONES DE LA VÍA CLÍNICA

\section{Indique código (COD) de la variación según CóDIGOS al final de la hoja}

\begin{tabular}{|l|l|l|l|l|}
\hline Fecha y turno & Variación y causa & Código & Acción tomada & Firma \\
\hline & & & \\
\hline & & & \\
\hline & & & \\
\hline & & & \\
\hline
\end{tabular}

\section{CÓDIGOS DE VARIACIONES}

\section{A. VARIACIONES DEBIDAS A LA CONDICIÓN CLÍNICA DEL ENFERMO}
1. Fiebre
2. Hemorragia o sangrado por acceso vascular
3. Infección de hittman
4. Problemas de canalización de fístula arterio- venosa
5. Nauseas, vómitos
6. Dolor no controlado
7. Fístula arterio-venosa no funcionante
8. Bacteriemia asociada a catéter IV
9. No tolerancia a sesión de hemodiálisis
10. Otras. Especificar

\section{B. VARIACIONES DEBIDAS AL PERSONAL SANITARIO}

11. Petición de pruebas no planificadas en la vía

12. Medicación pautada no planificada en la vía

13. Otras. Especificar

\section{VARIACIONES DEBIDAS A LA INSTITUCIÓN}

14. Retraso en el laboratorio

15. Retraso en Radiología

16. Otras. Especificar

\section{SALIDA DE VÍA CLÍNICA: $\square$ SI $\square$ N0}

CAUSA:

Tabla 2. Hoja de variaciones de la vía clínica 
[ M. Navarro Bermúdez ]

Desarrollo e implementación de una vía clínica de los pacientes que inician la hemodiálisis de forma programada

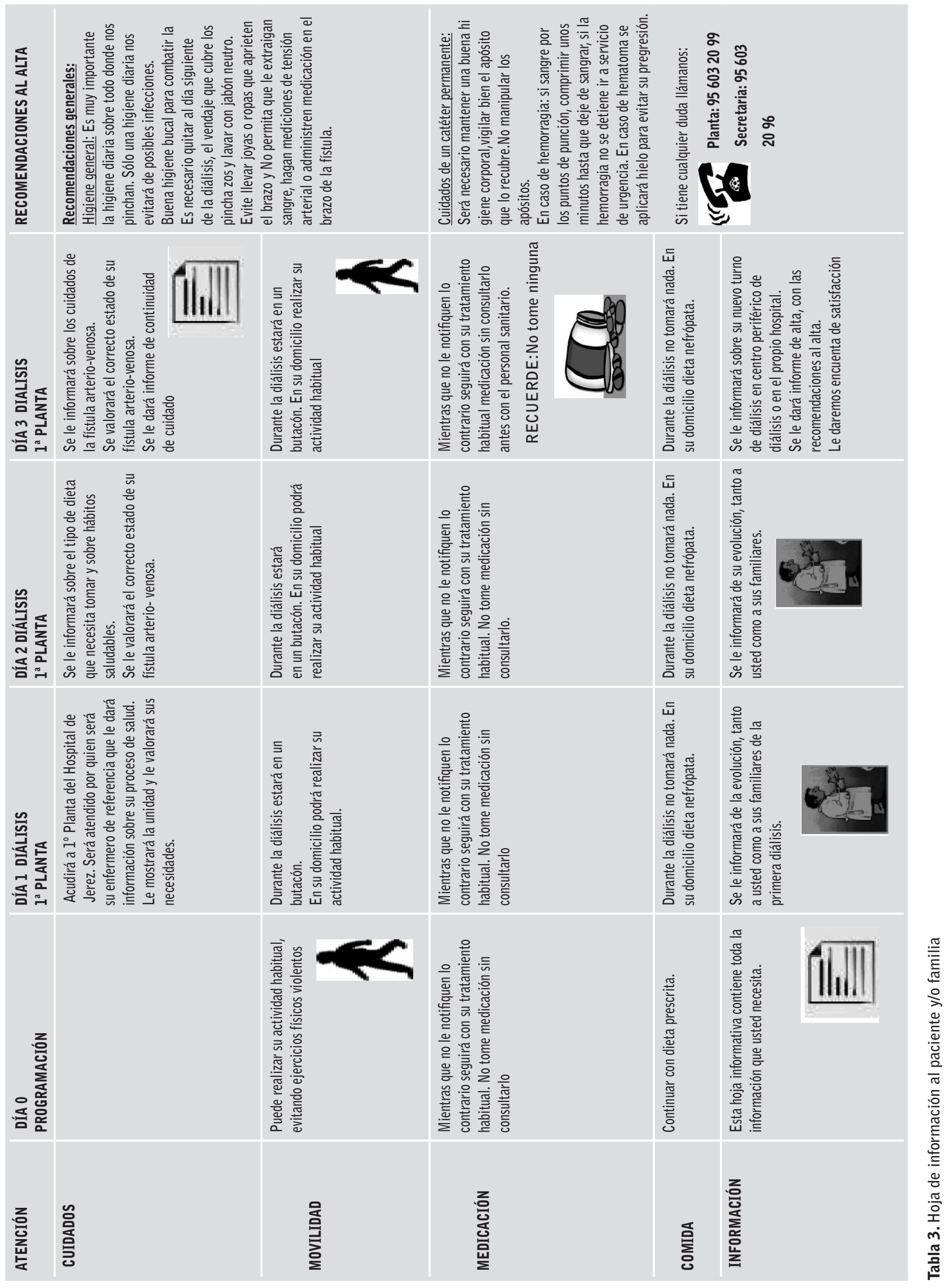


Otros problemas como serían los sesgos de población, no los vamos a tener en cuenta, ya que, todos los usuarios que inicien la diálisis de forma programada en el Hospital de Jerez serán incluidos en la vía clínica, evitando así posibles problemas como en los estudios de Stratta et $\mathrm{al}^{12}$ o Holtzman et $\mathrm{al}^{13}$ que evaluaron en sus estudios sobre la implementación de una vía clínica, la eficacia de la intervención de los pacientes que eran tratados mediante una vía clínica frente a los que no, con los consiguientes problemas éticos que esto pueda conllevar.

Los profesionales del servicio se encuentran muy motivados con el desarrollo e implementación de esta vía clínica, en primer lugar porque supone un cambio en su práctica clínica, lo cuál va a traer consigo una atención de mayor calidad y en segundo lugar, porque todos los profesionales sanitarios del servicio tienen entre sus objetivos individuales el participar y velar por el buen funcionamiento de esta vía clínica.

Otro aspecto que puede facilitarnos el buen desarrollo de esta vía clínica es que contamos con el apoyo de los distintos centros periféricos de diálisis que tenemos concertados ya que, tenemos su compromiso, de facilitarnos los traslados de pacientes del hospital a centros periféricos en los tiempos que tenemos estimados.

Recibido: Noviembre 2010

Revisado: Noviembre 2010

Modificado: Diciembre 2010

Aceptado: Enero 2011

\section{Bibliografía}

1. Hernández Gutiérrez, S et al. Resultados e impacto de una vía clínica para trasplante renal tras un año de desarrollo. Rev Calidad Asistencial 2003; 18(1):9-19.

2. Molina Martínez, FJ et al. Vías clínicas. Fundamentos, descripción y posibles aplicaciones en Neurología. Neurología, 2003; 18 (8): 439-451.

3. Viana Alonso, A et al. Descripción y evaluación de las vías clínicas en una red de hospitales públicos. Rev Calidad Asistencial 2002; 17(5):267-72.

4. III Plan Andaluz de Salud 2003-2008. Servicio Andaluz de Salud Consejería de Salud. Junta de Andalucía.

5. II Plan de calidad del Sistema Sanitario Público de Andalucía 2005-2008. Caminando hacia la excelencia. Edición, Consejería de Salud. Junta de Andalucía.

6. Mira JJ, Aranaz J. La satisfacción del paciente como una medida del resultado de la atención sanitaria. Med Clin (Barc) 2000; 114 (Supl 3):26-33.

7. Proceso Asistencial Integrado: Tratamiento Sustitutivo de la Insuficiencia Renal Crónica: Diálisis y Trasplante Renal.

8. Contreras Abad, MD: Guía para pacientes con tratamiento de Hemodiálisis. Ed. Hospital Universitario Reina Sofía, S.A.S., Córdoba, 2001.

9. Sociedad Española de Nefrología. Normas de Actuación Clínica en Nefrología. Tratamiento Sustitutivo de la insuficiencia renal crónica: Tratamiento conservador. Madrid, 1999.

10. Gorriz JL, Sancho A, Pallardó LM. Significado pronóstico de la diálisis programada en pacientes que inician tratamiento sustitutivo renal. Un estudio multicéntrico español. Nefrología. Vol. XXII. $n^{\circ} 1$. 2002.

11. Valdés F, Pérez Fontán $M$, Rodríguez-Carmona $A$, García Falcón T: Comienzo del tratamiento con diálisis. Nuevas tendencias. En: Aljama P, Arias M, Valderrábano: Insuficiencia renal progresiva. Editorial Grupo Entheos, Madrid, 2000.

12. Stratta R, Cushing K, Frisbie K, Miller S. Analysis of hospital charges after simultaneous pancreas-kidney transplantation in the era of managed care. Transplantation 1997; 64:287-92.

13. Holtzman J, Bjerke T, Kane R. The effects of clinical pathways for renal transplant on patient outcomes and length of stay. Medical Care 1998; 36:826-34. 